\title{
A Survey on Real-Time Automated Gridlock Control System
}

\author{
Madhubala E \\ UG Student, \\ Department of Computer Science and Engineering, \\ S.A. Engineering College, Poonamallee - Avadi \\ Road, Veeraraghavapuram, Thiruverkadu Post, \\ Chennai -600077
}

\author{
Nandhini C \\ UG Student, \\ Department of Computer Science and Engineering, \\ S. A. Engineering College, Poonamallee - Avadi \\ Road, Veeraraghavapuram, Thiruverkadu Post, \\ Chennai-600077.
}

\begin{abstract}
The increase in number of vehicles has led to the need for a smart system which can efficiently handle the traffic congestion. In this paper we address traffic light control problem and also about "Image subtraction ", "Gabor wavelet filter" and "Neural network" algorithms to lessen the whole waiting time of vehicle in a city. Emergency vehicles are detected using GPS and ZIGBEE sensors. Finally, the whole concept is explained based on "Image Processing".
\end{abstract}

KEYWORDS- Image subtraction, Gabor filter wavelet, Neural Network, Zigbee, GPS.

\section{INTRODUCTION}

Image processing is a method used to develop and improve the image to acquire some valuable information. It takes the image as an input and the output may be an image or description of that image. There are two level of image processing analog and digital. Analog method is used for processing the hardcopy of an image, while digital processing is carried out in digital photos using computers. "The digital technique has three notches

- $\quad$ pre-processing

- $\quad$ enhancement and display

- information extraction "

ZigBee is a wireless sensor. It is also simple and cheaper compared to other devices such as Bluetooth and WI-FI. These sensors are most widely used in an application which needs the long lasting battery and secure network. ZigBee can broadcast data for a distance of about 10 to $100 \mathrm{~m}$. It also ensures authentication and data integrity. They are robust in nature and also one can add and remove nodes whenever required.

The time and frequency domain's standard deviation artifacts will be reduced by the wavelet property. Gabor wavelet eliminates the uncertain information.

"The neural network is a computing system made up of a number of simple, highly interconnected processing elements, which process information by their dynamic state response to external inputs". These networks are typically organized in layers. Layers are made up of a numeral interconnected 'nodes' which confine an 'activation function'. Patterns are open to the

\section{Madhubala M \\ UG Student,}

Department of Computer Science and Engineering, S.A.Engineering College,Poonamallee Avadi Road, Veeraraghavapuram, Thiruverkadu Post, Chennai -600077 .

\author{
Prasanna Kumar R \\ Associate Professor, \\ Department of Computer Science and Engineering, \\ S. A. Engineering College, Poonamallee - Avadi \\ Road, Veeraraghavapuram, Thiruverkadu Post, \\ Chennai-600077.
}

system via the 'input layer', which leads into to one or more 'hidden layers' where the tangible processing is made via a system of biased 'connections'. The hidden layers then associate to an 'output layer'. Most Artificial Neural Network(ANNs) enclose some form of 'learning rule' which fluctuate the weights of the links according to the input's configuration that it is accessible with. Once a neural network is 'trained' to a reasonable intensity it may be worn as a diagnostic tool on other statistics.

\section{SURVEY ON AUTOMATIC TRAFFIC LIGHT CONTROL SYSTEM}

Manjunath. N and Nikschal. V.M[1] proposed controlling traffic automatically and identifying emergency vehicles using (Radio Frequency Identification Tag) RFID tag, RFID reader, and (Global System For Mobile Communication)GSM to send a message. RFID tags are used because of their longer lifetime and are used to identify objects. Communication between systems is done using (Universal Asynchronous Receiver/Transmitter) UART and RS-232. RFID tags are placed inside the ambulance. By matching the unique code which is already stored in the database the ambulance is found. Using GSM a message "EMERGENCY" will be sensed at the traffic intersection when an emergency vehicle is found. Once the message has been received the signal changes to green and remains the same until the vehicle crosses the signal.

Gayathri. R and Amudha. A [2] proposed the traffic control system that employs solar energy. The incessant power supply is prearranged by the photovoltaic system to the structure that uses (Radio Frequency) RF decoder, RF encoder, Photodiode and microcontroller. The photodiode and (Infrared) IR Sensors in the model are employed to perceive the traffic solidity in the signals which are in the line of sight configuration across the road. This system urges the emergency vehicle to trigger an alert message by the RF transmitters placed in the emergency vehicles and RF receiver placed in the junction. The purpose of solar panel in the circuit is to provide a clean source of energy to run the traffic light signal and to charge the battery during the daytime. Thus this method reduces the manpower and consumption of fuel. 
Divya. P and GunaSekhar. A.M [3] proposed how an emergency vehicle is allowed to change the signal to green at the time of its arrival. The signal in the other three way (four-way) will change to red. This system uses a raspberry pi microcontroller, several switches (north, south, east, west), an embedded system as a personal computer. Here the ambulance in particular lane should press a direction so that the traffic signal changes to green for 7 sec in that lane. So that the traffic congestion could not possibly happen in that particular lane, saving the life indeed.

VeeraVenkatesh and NazneenSyed[4]proposed a methodology for the clearance of emergency vehicles like ambulance and fire trucks from waiting in the traffic lane. Various edge detection and object counting methods are used to analyze the images from the camera efficiently. Here the edge detection technique detects the edges of the objects so we can easily identify the emergency vehicle. The object counting counts the frames and subtracts the items in the captured images. Unless the emergency vehicles cross the traffic lane the traffic light glows green, once it has crossed the lane it follows the previous pattern of the traffic signal.

Dr. Balamurugan. A, Navin Siva Kumar. G RajThilak. S and Selvakumar. P [5] projected an IOT based system which is expected to offer advanced connectivity of devices, systems, and services that go beyond machine to machine communications and shields a variety of protocols, domains, and applications. This paper mainly discusses about communication between the ambulance and various devices such as traffic signals and computers at hospitals. Here, the traffic signal is automatically controlled using a simple mobile phone app which uses GPS by capturing the latitude and longitude of the ambulance vehicle and dispatches a signal to the cloud system wherein the traffic signals are controlled by the cloud server. Thus this system provides a solution to reduce the emergency response time and avoids congestion of traffic.

BhargaviYadav. N, Mohan Kumar Naik. B [6], proposed a system where each vehicle is fixed with a RFID tag and the emergency vehicle are equipped with a ZigBee transmitter to differentiate with the normal vehicles. The duration of the green light is decided on the number of vehicles that are within the radar range of the RFID transceiver. The time period of the green signal will be around 30 seconds if the vehicles range between 10 to 20 . If there are less than 5 vehicles the duration would be around 10 seconds. The less, the vehicles present more will the duration of the red light. During the emergency situation, the ZigBee sends the unique ID code to the ZigBee receiver equipped at the traffic junction. The system inspects the unique ID with the codes stored already and turns the light to green till the ambulance crosses the lane.

Vidhya. K, BazilaBanu. A [7] has proposed a system that automatically controls the traffic using image processing technique. Here the trapped images are processed and transferred into grayscale images and the vehicle count is detected by contouring technique. This paper also includes the count of stationary vehicles. Modifications in signal timings are done using a raspberry pi microcontroller. Here image processing technique is done using open source software called OpenCV system.

MaramBaniYounes[8] has proposed Intelligent Traffic Light Controlling(ITLC) algorithm. This methodology considers the real-time traffic pour that intends to cross the road roundabout of interest, at the same time as setting up the time phrases of each traffic light. The data structure intends at escalating the traffic glibness by declining the waiting time of the vehicles at the signalized road intersections. For various simulated circumstances, the authors contrast the performance of the specified ITLC data structure to the earlier methods used. The increase in percentage is of nearly $25 \%$ in contrast to the earlier traffic light schedules where the specified structure reduces the queuing delay along with the increase in clearance of the traffic.
Kavya. G and Saranya. B[9] has proposed system works based on a PIC16F8 series microcontroller. Traffic-light signals are enhanced based on the congestion on road at that moment. With the aid of transistors IR sensors are connected to the microcontroller and these sensors detect the solidity of the vehicles within a fixed distance. The sensors provide priority to green light that glows for a longer time followed by other levels. The side with the next priority level follows the first priority level. Here the software's such as MPLAB \& CCS C Compiler and the language such as Embedded $\mathrm{C}$ or Assembly are used. Based on the timing allotted the density of vehicle is classified into three zones: Low, Medium, and High based accordingly. Hence, the traffic light is controlled based on the compactness of the vehicle.

Anshul Gupta, ChahatAhuja, Apar Vijay, Shipra, PranjalKatara[10] describes how the one-way tunnel could work without collision of the vehicles from either way. The four wheeler vehicle is recognized once it passes through the pressure transducer, which shows an electric signal corresponding to its weight. The infrared proximity sensor senses the vehicle and increases the count of the integer programming counter algorithm, which will be decreased only when the vehicle comes out through the other side of the tunnel fixed with IR sensors similar to the entrance. If the IR senses that the vehicle entered has not come out yet, it changes the traffic light at both the ends to red. This will stop vehicles further to enter the tunnel. The MCU is responsible for sending alerts about the vehicle stuck in the tunnel, to the security room or the guard of the tunnel. The traffic light turns to yellow if there is a two wheeler vehicle inside the tunnel.

Nidhi. D Agarwal, AmitShau[11] has proposed that traffic can be controlled using Image Processing technique even in shadows and various lightning conditions. It makes the image more precise by brightening, blurring, and image enhancement. This paper also explains the effective use of image restoration. It also explains about an algorithm for detecting the emergency vehicle. Since this process requires only cameras and sensors hence it is cost effective.

Royani. T, Haddadina. J, and Alipoor. M [12] has proposed the use of a fuzzy neural network for managing traffic light signals. The goal of this paper is to augment the vehicular throughput and reduce delays. Using fuzzy classifying traffic flow at intersections, the system stores the stream of traffic and is stocked with a set of rules into the knowledge base. Based on the distortion in the traffic flow, the rules are updated using a neural network. Decisions are made using a fuzzy logic controller. Fuzzy logics are used to make decisions with incomplete information. Thus this paper effectively explains the use of fuzzy neural network and knowledge base in controlling traffic lights with better performance and adaptability.

Osigwe Uchenna Chinyere, Oladipo Onaolapo Francisca and Onibere Emmanuel Amano[13] designed the controller using the fuzzy rules by determining the physical state and the solidity of the road. The hybrid system is designed by combining two systems where the counter queue is increased as the vehicle waiting to pass the junction is more.

Muhammad Arshad Khattak[14] has proposed a system that senses the presence or absence of vehicle within a certain range by setting the appropriate duration for the traffic signals to react accordingly. This system consists of three vital chunks, PLC controller, Hardware, which typically comprises of red, yellow and green lights and the final part is the sensor. The existence of the vehicle is patterned by sensors and the sensor is checked by PLC. Based on the priorities it affords signal to the traffic light poles for ON or OFF the red, yellow or green lights and ON time depends on the specific priorities once the infrared object detector picks up the presence of a car, a switch causes the light to change. Thus this system effectively performs traffic management. 
NasriSulaiman, ZeyadAssi, Obaid, Marhaban. M.H, and Hamidon. M.N [15] has proposed that the state of the art of Field Programmable Gate Array(FPGA) with the focus on FPGAbased systems. This paper mainly focuses on the main differences between software-based control systems with respect to FPGA-based systems, and the main features for FPGA technology and its real-time applications. Programming is done in FPGA using a language called hardware description language.

\section{PROPOSED SYSTEM}

In this proposed work we demonstrate two modules:

(i) Signal timing variant based on vehicle occupancy.

(ii) Advanced ambulance signaling.

The first part that describes the alteration in the timing of each signal based on the vehicle occupancy in the corresponding road. This process also shows the video which is captured from the top view that is mounted on the traffic junction of the consequent road. The empty lane's image is considered as a reference picture. Hence, the real-time image of the corresponding road is compared with the reference image to obtain the subtracted image.

Gabor Filter: In FFT, only the frequency information is highlighted whereas the spatial information of the image is neglected. Here, the high frequency represents the presence of an object. We need the Gabor wavelet to establish both the spatial and frequency information due to the lack of spatial information which makes the recognition to be inaccurate.

Neural network: By using the neural network we can monitor the total occupancy based on both image processing and Gabor filter. We use feed forward, back propagation, a neural network to get the vehicle occupancy ratio. If it is found to be low then the signal time is decremented.

Advanced ambulance signalling: Ambulance is fitted with a device that acquires the geo-locality by the means of GPS. When the user switches ON, it broadcasts its GPS data through the Zigbee transmitter.

When the Zigbee signal of the ambulance is received by the Zigbee receiver at the traffic junction, it ensures whether the ambulance is in range with the traffic signal if so, the signal time is altered and the green signal glows in the path where the ambulance moves.

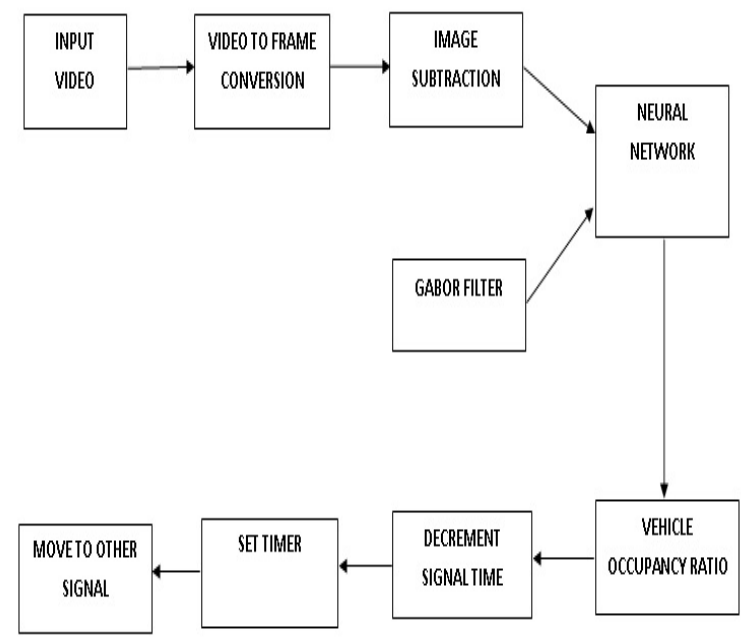

Figure 3.1 Traffic signal timing variant based on vehicle occupancy.

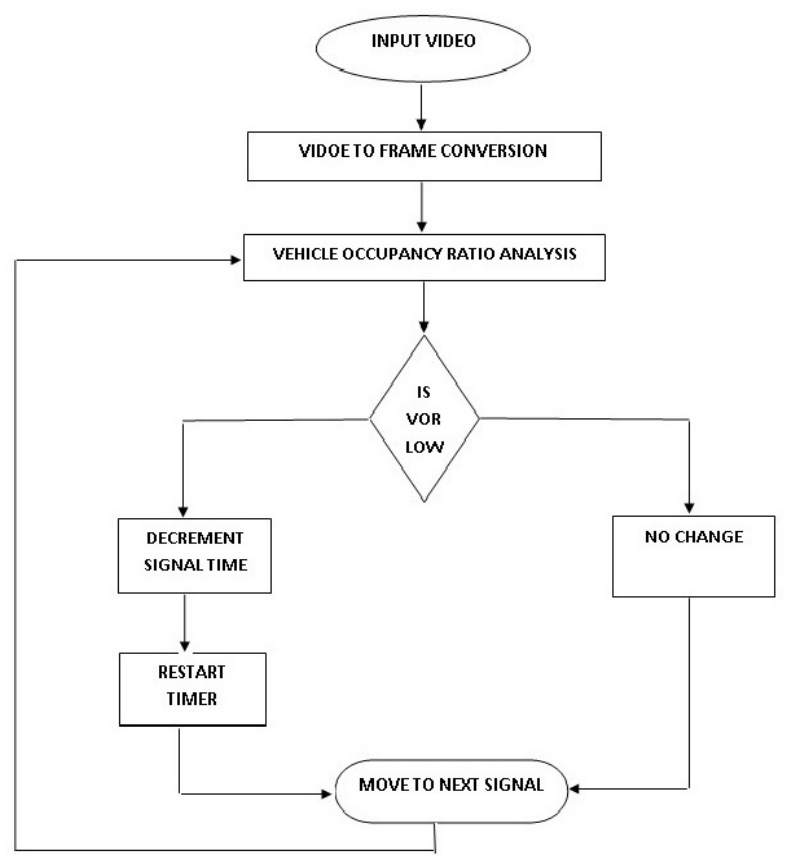

Figure 3.2 Flowchart

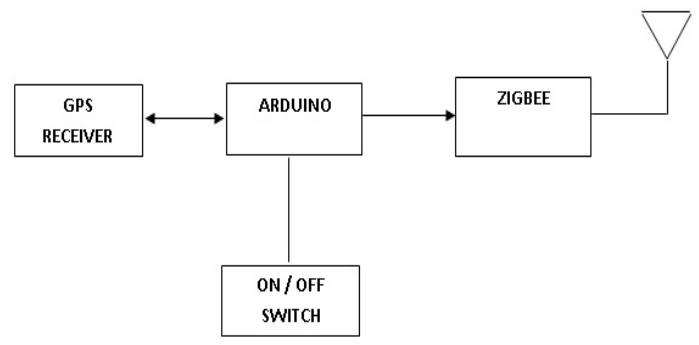

(a)

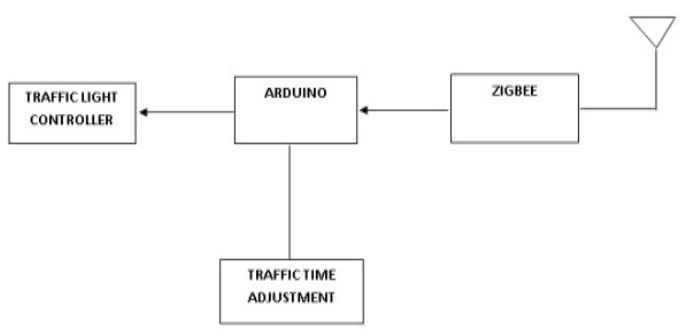

(b)

Figure 3.3 Advanced ambulance signalling system

(a) Ambulance (b) Signal

\section{CONCLUSION}

This paper presents a survey on monitoring traffic signal and has the ability to retract the signal time of the traffic based on the vehicle occupancy. The above-stated works on different titles have astounded the imperfections in Gabor technique, Image subtraction and neural network thereby providing redundancy in error to an extent and also accuracy is achieved here. The shortcomings of image processing will still be a future challenge 


\section{A Survey on Real-Time Automated Gridlock Control System}

in forthcoming years. Hence, the benefits of this technology will be a major clarification for the image processing methods.

\section{REFERENCES}

[1] Manjunath N, Nikschal V.M, SurabhiMohta, Sindhuja N, " Design of an automated traffic control system for emergency vehicle clearance", In International Journal of Emerging Research in Management \&Technology(2016), Page No.278-281.

[2] Gayathri. R and Amudha. A "Solar Powered Traffic Control System Based on Traffic density with Emergency Vehicle Alert", In Middle-East Journal of Scientific Research 24 (2): 234-238, ISSN 1990-9233(2016), Page No. 234-238.

[3] Divya P, GunaSekhar A.M, "Innovative Congestion Control System for Ambulance Using Raspberry Pi", In International Journal and Magazine of Engineering, Technology, Management and Research(2016), Page No. 586-590.

[4] VeeraVenkatesh, Nazneen Syed,"Smart traffic control system for emergency vehicle Ponamallclearance", InInternational Journal of Innovative Research in Computer and Communication Engineering(2015), Page No.7242-7246.

[5] Dr. Balamurugan. A, Navin Siva Kumar. G, Raj Thilak. S, Selvakumar. P, " Automated Emergency System in Ambulance to Control Traffic Signals using IoT", In International Journal of Engineering And Computer Science ISSN:2319-7242(2015), Page No. 11533-11539.

[6] BhargaviYadavN, B Mohan Kumar Naik, "RFID and ZIGBEE based intelligent traffic control system", In InternationalJournal of Computer Engineering and Applications (2016), Page No. 411-417.

[7] Vidhya K, BazilaBanu, "Density-based traffic signal system", In International Journal of Innovative Research in Science, Engineering, and Technology(2014), Page No. 2218-2983.

[8] MaramBaniYounes, AzzedineBoukerche, "An Intelligent Traffic Light Scheduling Algorithm Through VANETs", In 10th IEEE International Workshop on Performance and Management of Wireless and Mobile Networks(2014), Page No. 637-642.

[9] Kavya. G, Saranya. B, "Density-Based Intelligent Traffic Signal System Using PIC Microcontroller", In International Journal of Research in Applied Science \& Engineering Technology (IJRASET)(2015), Page No. 205-209.

[10] Anshul Gupta, ChahatAhuja, Apar Vijay, Shipra, PranjalKatara, " Automated and Intelligent Tunnel Traffic Control System", In International Journal of Emerging Technology and Advanced Engineering(2014), Page NO. 532-536.

[11] Nidhi D Agarwal, AmitSahu, "Intelligent real-time traffic controller using Image Processing ", In International Journal of Science and Research(2013), Page No. 29802983.

[12] Royani T, Haddadina J, Alipoor M, "Control Of traffic light in isolated intersection using fuzzy neural network and genetic algorithm", In International Journal of Computer and Electrical Engineering(2013), Page No.142146.

[13] OsigweUchennaChinyere,

OladipoOnaolapoFrancisca,Onibere Emmanuel Amano, "Design and simulation of an intelligent traffic control system", In International Journal of Advances in Engineering \& Technology(2011), Page No.47-57.
[14] Muhammad ArshadKhattak " PLC Based Intelligent Traffic Control System", In International Journal of Electrical \& Computer Sciences IJECS-IJENS(2011), Page No.69-73.

[15] NasriSulaiman, ZeyadAssiObaid, M. H. Marhaban and M. N. Hamidon,"Design and Implementation of FPGA-Based Systems", In Australian Journal of Basic and Applied Sciences(2009), Page No.3575-3596. 